Neurosurg Focus 19 (3):E7, 2005

\title{
Pregnancy-related vertebral hemangioma
}

\author{
Case report, review of the literature, and management algorithm
}

\author{
John H. Chi, M.D., M.P.H., Geoffrey T. Manley, M.D., Ph.D., and Dean Chou, M.D. \\ Department of Neurological Surgery, San Francisco General Hospital, University of California, San \\ Francisco, California
}

\begin{abstract}
Pregnancy is a recognized risk factor for quiescent vertebral hemangiomas becoming symptomatic; this usually occurs during the 3rd month of gestation. The natural history of these lesions is poorly understood, and treatment practices must consider the overall safety of the mother and fetus. The authors report a case of cervical vertebral hemangioma presenting during the 24th week of pregnancy and review the current literature.

A 26-year-old woman in her 24th week of pregnancy presented with upper-back pain and progressive spastic paresis in the legs. Neuroimaging studies revealed a diffuse C-7 vertebral body lesion with extradural extension and compression of the spinal cord consistent with a vertebral hemangioma. Successful decompression was accomplished, and the fetus experienced no adverse effects from the surgery.

In a review of the literature, 23 cases of pregnancy-related vertebral hemangioma dating back to 1927 were identified. Prepartum surgical decompression was performed in eight patients, postpartum surgery was performed in 12, and surgery was not performed in four. Overall, patients experienced excellent neurological recovery, regardless of the severity and duration of spastic paresis.

Observation should be considered for symptomatic patients at greater than 32 weeks gestation. Surgery should be considered for patients with severe neurological deficits at less than 32 weeks of gestation.
\end{abstract}

\section{KEY WORDS • pregnancy - vertebral hemangioma • myelopathy • anterior corpectomy}

Vertebral hemangiomas are benign vascular lesions of the vertebral column that occur in 10 to $12 \%$ of the general population and become symptomatic in $1 \%$ of those afflicted. Pregnancy is a recognized risk factor coinciding with the development of a rapid onset of symptoms from these normally asymptomatic lesions. Symptoms most often occur during the third trimester of pregnancy and most commonly affect the thoracic spinal levels, beginning as radicular or back pain and leg paresthesia, and progressing to spastic paresis with incontinence within weeks.,8,33,34 $\mathrm{Di}$ agnostic imaging reveals compression of the spinal cord from a vertebral and/or extradural hemangioma. Treatment of these lesions requires consideration of multiple factors, including the location of the spinal cord compression, the speed of neurological decline, the stage of pregnancy, and the potential risks to the fetus from various treatment modalities. The natural history of pregnancy-related vertebral hemangiomas is poorly understood, and no management algorithm is currently available to help direct the timing of surgical treatment.

In this report we describe a patient with a symptomatic cervical vertebral hemangioma with extradural extension that was treated during pregnancy by anterior corpectomy

Abbreviations used in this paper: $\mathrm{CT}=$ computerized tomography; MR = magnetic resonance. and fusion. We also review the current literature on pregnancy-related spinal hemangiomas and propose a treatment algorithm based on the stage of pregnancy.

\section{CASE REPORT}

History and Examination. This 26-year-old obese women, gravida 1 , para 0 , in her 24th week of pregnancy presented with a 3-week history of upper-back pain, leg paresthesia, progressive difficulty walking, and leg weakness. She did not have headaches, fevers, chills, or incontinence. She had received routine prenatal care, and her pregnancy was unremarkable until onset of symptoms indicative of a hemangioma. On evaluation, she exhibited profound myelopathy with 3/5 motor strength in the legs, a T-10 sensory level, and hyperreflexia with sustained clonus in the legs. Examination of the arms revealed only slight intrinsic muscle weakness of the left hand.

Neuroimaging. Admission MR imaging of the spine revealed an isolated C-7 vertebral body lesion with extradural extension and compression of the spinal cord, and evidence of $\mathrm{T}_{2}$ signal abnormality within the cord (Fig. 1A and D). Gadolinium contrast material was not administered because of the patient's pregnancy. The lesion appeared to expand the entire vertebral body, but did not involve adja- 

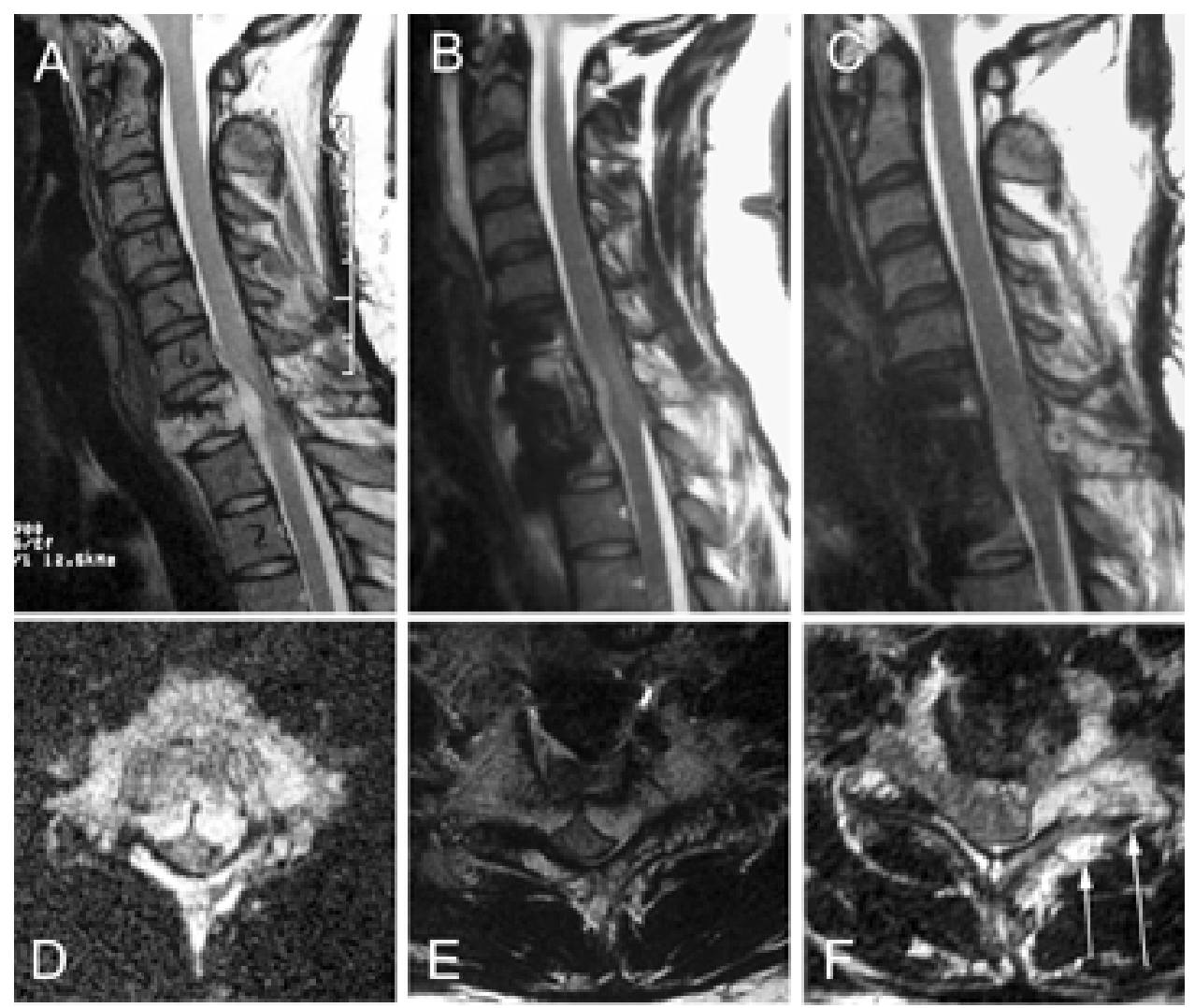

Fig. 1. A-C: Sagittal $\mathrm{T}_{2}$-weighted noncontrast-enhanced cervical spine MR images obtained preoperatively (A), on Day 3 postoperatively (B), and 2 weeks postpartum (4 months postoperatively, C). D-F: Axial postoperative $\mathrm{T}_{2}$-weighted noncontrast-enhanced MR images through the $\mathrm{C}-7$ vertebral body ( $\mathrm{T}_{2}$-weighted fat-suppressed sequence, $\left.\mathrm{D}\right)$, on Day 3 postoperatively (E), and 2 weeks postpartum (4 months postoperatively, F). Improvement of ventral epidural mass effect is seen postsurgery, with progression of paravertebral hemangioma (arrows).

cent disc spaces or paravertebral soft tissues. Admission CT scanning revealed a honeycomb-patterned C-7 vertebra and large vacuolated spaces within the entire spinal column, including posterior elements (Fig. 2). These findings were consistent with a vertebral hemangioma.

Initial Treatment. The patient was initially given glucocorticoid boluses (4 mg Decadron intravenously every 6 hours) and remained stable for 3 days on bedrest. On Day 4 of her hospitalization, she experienced a decline in motor function, prompting emergency decompression. Repeated MR imaging performed at that time revealed no changes in the lesion.

Operation. Anterior cervical corpectomy was performed through a wide, low-anterior neck dissection. Care was taken to preserve the right recurrent laryngeal nerve. Continuous fetal heart monitoring was performed by an obstetric nurse during surgery. There were no signs of fetal distress during the operation. Extensive bleeding was encountered during corpectomy; an estimated blood loss of approximately $2.5 \mathrm{~L}$ necessitated transfusion of $2 \mathrm{U}$ of blood. Corpectomy at C-7 was achieved and osseous bleeding was easily stopped with bone wax. Extradural hemangioma tissue overlying the dura mater was partially removed, but extensive bleeding prevented complete resection. Cadaveric tibia allograft was used for a bone graft, and standard anterior cervical plate stabilization was performed. Several specimens were obtained intraoperatively and sent to the Department of Pathology for histological examination. No fluoroscopy was used during the operation.

Histological examination demonstrated fragments of bone with prominent vascular channels, vascularized fibrous tissue, and benign vascularized fibroadipose tissue, which were consistent with a diagnosis of osseous hemangioma. Postoperative MR imaging demonstrated subtotal resection of the hemangioma and persistent extradural compression of the spinal canal (Fig. 1B and E).

Postoperative Course. After surgery her paresthesias resolved and motor strength in both legs improved to $4+/ 5$ within 1 week. At the time of discharge, the patient exhibited hyperreflexia and clonus in the lower extremities but was ambulating with a walker. There were no complications regarding her pregnancy at any time during her stay in the hospital.

At 40 weeks gestation, the patient delivered a healthy baby by normal spontaneous vaginal delivery. The baby's Apgar score was 8 and 9 and its weight was $3420 \mathrm{~g}$. By the time of delivery, the patient was ambulating independently and had attained full strength in her legs, normal bowel and bladder function, and resolution of pain. Residual weakness and numbness of the left hand was minimal and did not interfere with her normal daily activities. Postpartum MR imaging demonstrated a decrease in extradural spinal com- 


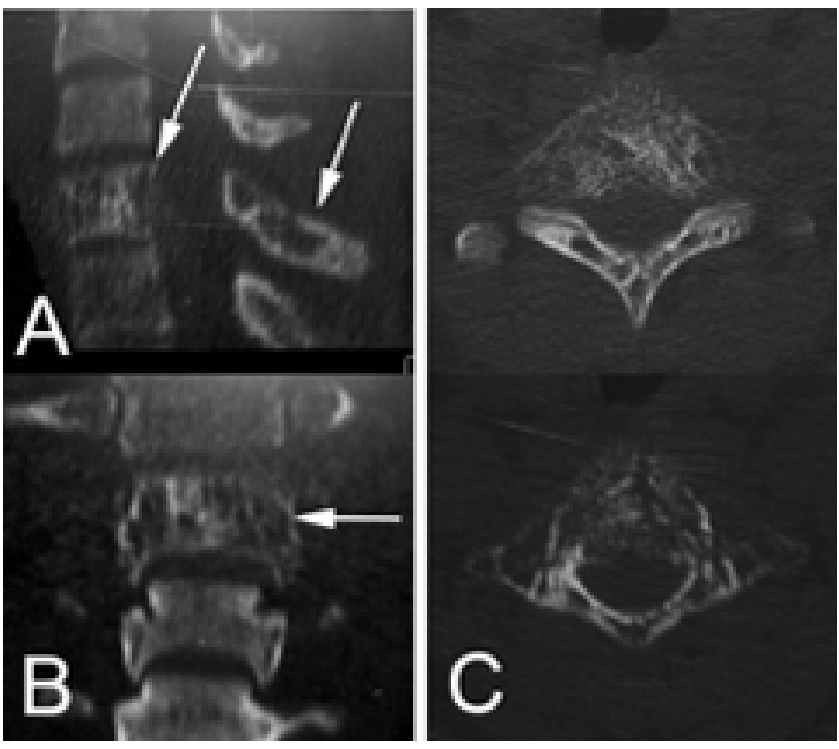

Fig. 2. Preoperative CT scans with reconstruction of the lower cervical spine in sagittal (A), coronal (B), and axial (through the C7 vertebra, C) views demonstrating a classic honeycomb pattern involving the entire C-7 vertebra, consistent with hemangioma (arrows).

pression, but extension of the hemangioma into the paravertebral space (Fig. 1C and F) was seen. A cervical X-ray film demonstrated a stable fusion graft and normal spinal alignment (Fig. 3).

\section{REVIEW OF THE LITERATURE}

A review of the literature was conducted by searching PubMed (1950-2005) for the key words "pregnancy," "hemangioma," "spine," and "vertebrae" and manually searching references in the bibliography of relevant articles published prior to 1950 . Only manuscripts or abstracts written in the English language were reviewed. Cases from the literature were included if the patients were found to have a spinal hemangioma affecting vertebrae and/or involving the extradural space and were pregnant at the time of diagnosis. Patients were excluded if the timing of their pregnancy was remote to symptomatic presentation $(>2$ months) and if the lesion was described as intradural.

Twenty-three cases of pregnancy-related vertebral hemangioma were identified (Table 1). As noted by Fields and Jones, ${ }^{14}$ the first case was reported in 1927 by Balado, whereas half of the cases reviewed were published after 1980. The mean patient age at presentation was 29 years. Eleven women had had previous pregnancies; $1,6,9,14,19,21,26,34$, 35,37 six of these women had experienced mild neurological symptoms during prior pregnancies that had resolved after parturition. ${ }^{14,19,21,34,35,37}$ For nine patients it was a first-time pregnancy. $2,11,14,23-25,36$ The mean period of gestation at presentation was 7.65 months. Two patients presented after childbirth. ${ }^{35,36}$ Abrupt onset of pain followed by paresthesias and onset of spastic paresis within weeks occurred in most cases. The mean duration of symptoms before treatment or delivery was 7.6 weeks.

Prepartum surgery was performed in seven patients, 2,19 ,

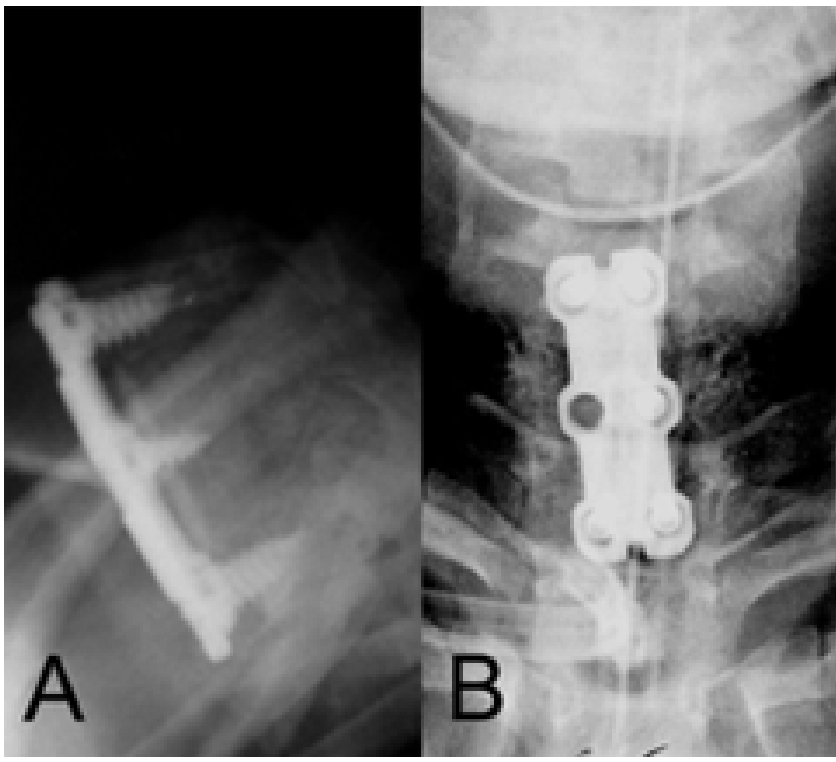

Fig. 3. Postoperative $x$-ray films revealing lateral swimmer's (A) and anteroposterior (B) views of the cervical spine 4 months postoperatively.

23-26,34 postpartum surgery in $12,8,9,11,12,14,21,23,26,35-37$ and no surgery in four. ${ }^{5,6,14,26}$ Among patients treated surgically before childbirth, four experienced preterm labor,:2,23,25,26 prepartum surgery was also associated with two maternal deaths ${ }^{19,26}$ and two fetal deaths, ${ }^{23,25}$ but all occurred prior to 1985 . Prepartum embolization without surgery was reported in one case as producing good neurological recovery; ${ }^{5,6}$ however, long-term follow-up data have not been published. Among patients treated surgically after childbirth, two improved following delivery, ${ }^{26}$ two experienced transient improvement followed by decline and persistent deficit, ${ }^{14,21}$ and five deteriorated or had persistent deficits after delivery. ${ }^{26,33,35-37}$

Excluding deaths, all patients demonstrated excellent neurological recovery, independent ambulation, and resolution of pain at follow-up review (range 4 months-5 years). Mild paresthesia and weakness not interfering with activities of daily living were found in two patients. ${ }^{25,26} \mathrm{Ex}-$ cluding the two perinatal deaths, there were no reports of adverse effects on the infants, and no adverse effects were noted in the children during the follow-up periods reported. In the majority of patients treated with surgery, posterior approaches were preferred, mainly because of the predominance of thoracic-level lesions and the ease of posterior decompression. Anterior approaches, including one case of prepartum thoracotomy, ${ }^{24}$ were used in three patients; $;{ }^{24,33,35}$ none led to surgery-related morbidity. Revision surgery for worsening symptoms after the initial surgery was performed in two patients. ${ }^{34,37}$

\section{DISCUSSION}

Vertebral hemangiomas are the most common primary spinal neoplasm and are present in 10 to $12 \%$ of the population, based on autopsy studies performed in adults. . $7,29,31,37$ Usually incidental, asymptomatic, and solitary, these are benign vascular lesions that can give rise to symptoms in 
TABLE 1

Literature review of 23 pregnancy-related vertebral hemangiomas*

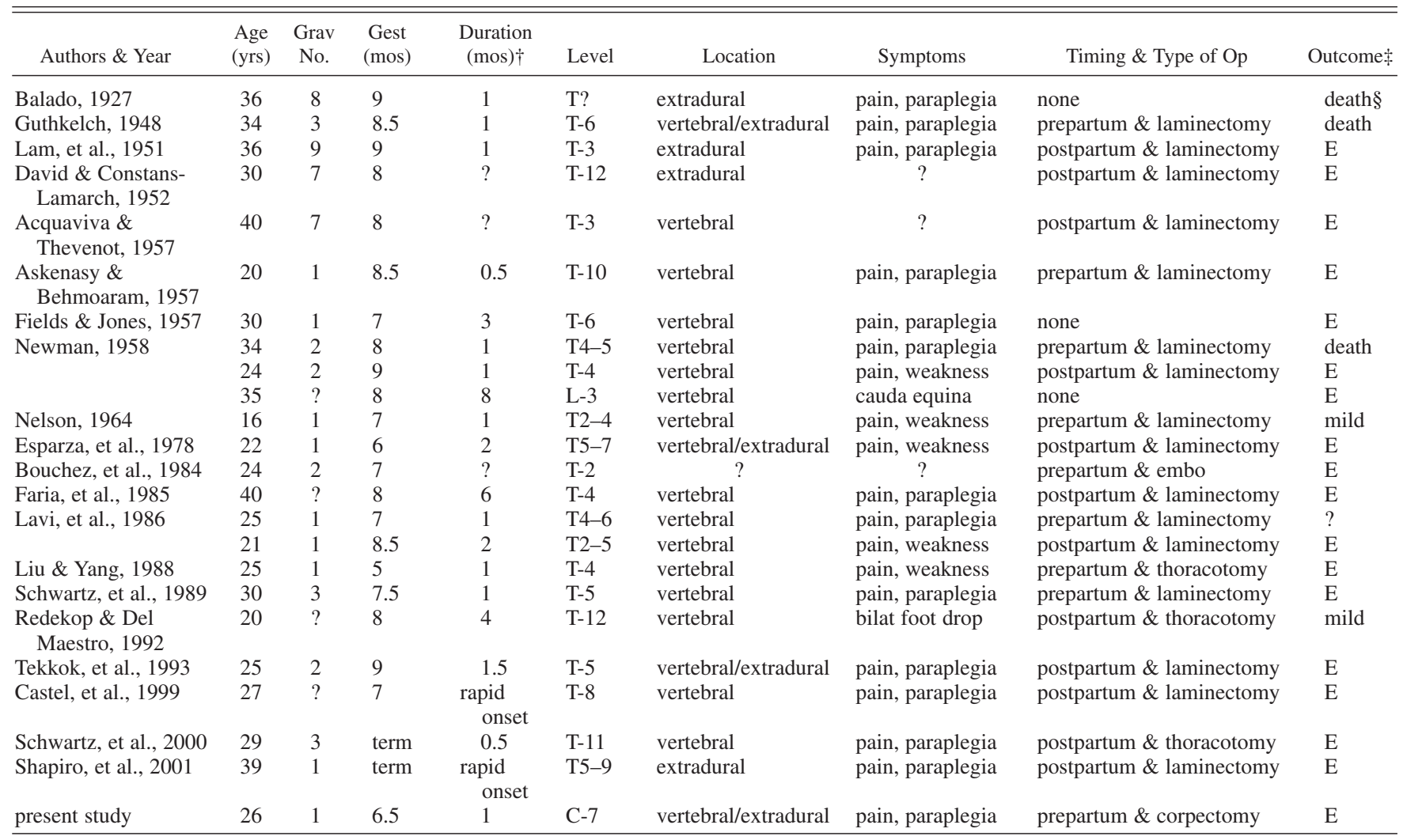

$* \mathrm{E}=$ excellent recovery with independent ambulation and no disability at follow-up evaluation; embo = embolization; gest = gestation; grav = gravida; mild $=$ residual weakness or foot drop, or assisted ambulation with cane or walker at follow-up evaluation; ? = not reported.

$\dagger$ Duration of symptoms was determined based on the interval from onset of the first symptoms reported by the patient to the time of diagnosis or parturition.

\$ Maternal outcomes only.

$\S$ Refers to death without surgery/treatment and prior to parturition.

rare circumstances. Symptoms include radicular pain in most cases, and neurological compromise can occur in up to $40 \%$ of symptomatic cases. Symptoms are thought to develop by the following mechanisms: 1) vascular expansion of a vertebral body, pedicle, lamina, or facet leading to and with direct compression of nerve roots and/or the spinal canal; 2) subperiosteal extension of the hemangioma resulting in an extradural mass compressing the spinal cord; and 3) compression fractures and vertebral collapse secondary to replacement of bone with hemangioma. Rarely, a vertebral hemangioma may cause bleeding with epidural hematoma or vascular steal with spinal cord ischemia.

Vertebral hemangiomas exhibit a classic radiographic appearance of coarse vertical striations, referred to as a honeycomb pattern, on plain spine x-ray films or CT scans. Conventional MR imaging is less definitive for hemangioma, but the high blood content of the lesion demonstrates high $\mathrm{T}_{2}$-weighted signal intensity on fat-suppression sequences. Angiograms can also aid in diagnosis and provide a road map for treatment (that is, embolization). The vascular supply to vertebral hemangiomas usually originates from small branches of the intercostal or lumbar arteries that arise proximal to the radicular branches. Certain radiographic features such as neural arch involvement, complete vertebral body involvement, expanded osseous cortex with indistinct margins, an irregular honeycomb pattern of bone, an epidural mass, and fatty stroma are associated with the development of symptoms, ${ }^{22}$ which most pregnancy-related cases demonstrate.

The histological appearance of hemangiomas consists of benign vascular proliferation with normal capillary and venous structure. ${ }^{8,29,35}$ Two types have been described: cavernous or capillary. The most common type of vertebral hemangioma is the cavernous type, which is characterized by large sinusoidal spaces lined by a single layer of epithelium. The capillary type of vertebral hemangioma differs from the cavernous type only by having smaller vascular channels. Whereas asymptomatic hemangiomas occur primarily in the thoracolumbar spine, symptomatic hemangiomas most commonly occur at the thoracic level. ${ }^{27}$ Lumbar or cervical locations for symptomatic hemangiomas are exceedingly rare.

As detailed in the report by Fields and Jones, ${ }^{14}$ pregnancy-related symptomatic vertebral hemangioma was first described by Balado in 1927. Pregnancy is thought to induce symptoms by altering normal vascular hemodynamics, causing increased blood flow and volume in the hemangioma. Pregnancy itself leads to a 30 to $50 \%$ increase in 
blood volume when the gravid uterus begins to compress the vena cava during the third trimester. ${ }^{35,37}$ Venous obstruction and increased intraabdominal pressure lead to increased flow through the vertebral venous system and may provide enough stimulus for the expansion and growth of previously existing vertebral hemangiomas. ${ }^{25,37}$ Extradural extension usually occurs in the subperiosteal plane. ${ }^{10}$ Alternatively, endothelial proliferation stimulated by estrogen may result in mitogenic enlargement of hemangiomas during pregnancy. Nevertheless, estrogen and progesterone receptors seem to be absent in biopsy samples of pregnancy-related hemangiomas. ${ }^{35}$ The third trimester of pregnancy has been reported as the most common time for symptomatic presentation, but second-trimester and postparturition cases have also been reported. , $33,35,37^{-1}$

Treatment options for symptomatic hemangioma include radiation therapy, embolization, percutaneous sclerotherapy, vertebroplasty, and surgery. Radiation therapy is the treatment of choice for lesions causing pain only. Recent case series in which radiation therapy was used (20-40 Gy over 2-4 weeks) demonstrate a symptom improvement rate of up to $77 \%$,7,8,12,30,35 and include improvement in neurological conditions in patients presenting with deficits. Risks include delayed radiation-induced myelopathy, which occurs with doses greater than 45 Gy to 50 Gy. Pregnancy is a relative contraindication to radiation therapy, although newer stereotactic radiosurgery modalities may play a role in increasing the safety of radiation therapy during pregnancy. ${ }^{17}$ Embolization has been claimed in several case reports to improve symptoms in patients with pain and neurological deficits, ${ }^{3,4,11,20,28}$ but is typically used as an adjunct to surgery to aid in minimizing blood loss. ${ }^{38}$ Complications include vascular injury and radiation exposure to the fetus during fluoroscopy. No cases of prepartum embolization for vertebral hemangioma have been reported.

Percutaneous vertebral sclerotherapy and vertebroplasty is a promising new technique that seems to be efficacious in patients with both pain and neurological deficits, ${ }^{13,15,16}$, 18,32 but its use has yet to be reported in a pregnant patient. Surgery is the preferred treatment for patients with neurological deficits, especially with rapid onset of symptoms. The advantages of surgery include immediate and direct decompression of the spinal canal and avoidance of fetal exposure to excessive radiation. Disadvantages include significant intraoperative blood loss and the usual risks of surgery and anesthesia for a pregnant patient.

During pregnancy, treatment options have included the following: 1) induction of preterm delivery, which places the fetus at a nearly $50 \%$ risk of morbidity or mortality if gestation is earlier than 32 weeks; 2) expectant observation and postparturition treatment; or 3) prepartum surgery. Because of the aggressive nature of pregnancy-related vertebral hemangiomas, surgical decompression is usually subtotal. Incomplete resection has been reported to result in up to 20 to $30 \%$ recurrence rates, ${ }^{28}$ which argues that adjunct therapy is warranted as soon as it is safely possible. Some patients may experience spontaneous improvement in symptoms without treatment postpartum, which allows for less urgent management of the lesion. Future recurrence and deterioration is frequent, however, and argues for early postpartum intervention.

\section{MANAGEMENT ALGORITHM}

Although there is no Class I data to support an official recommendation or guideline, there does appear to be a trend based on a review of the cases available. Thus, an algorithm may be considered in the management of symptomatic vertebral hemangioma in pregnancy (Fig. 4). For patients at 36 weeks of gestation or later, expectant management with observation can be considered, given that these patients have historically recovered well after delivery

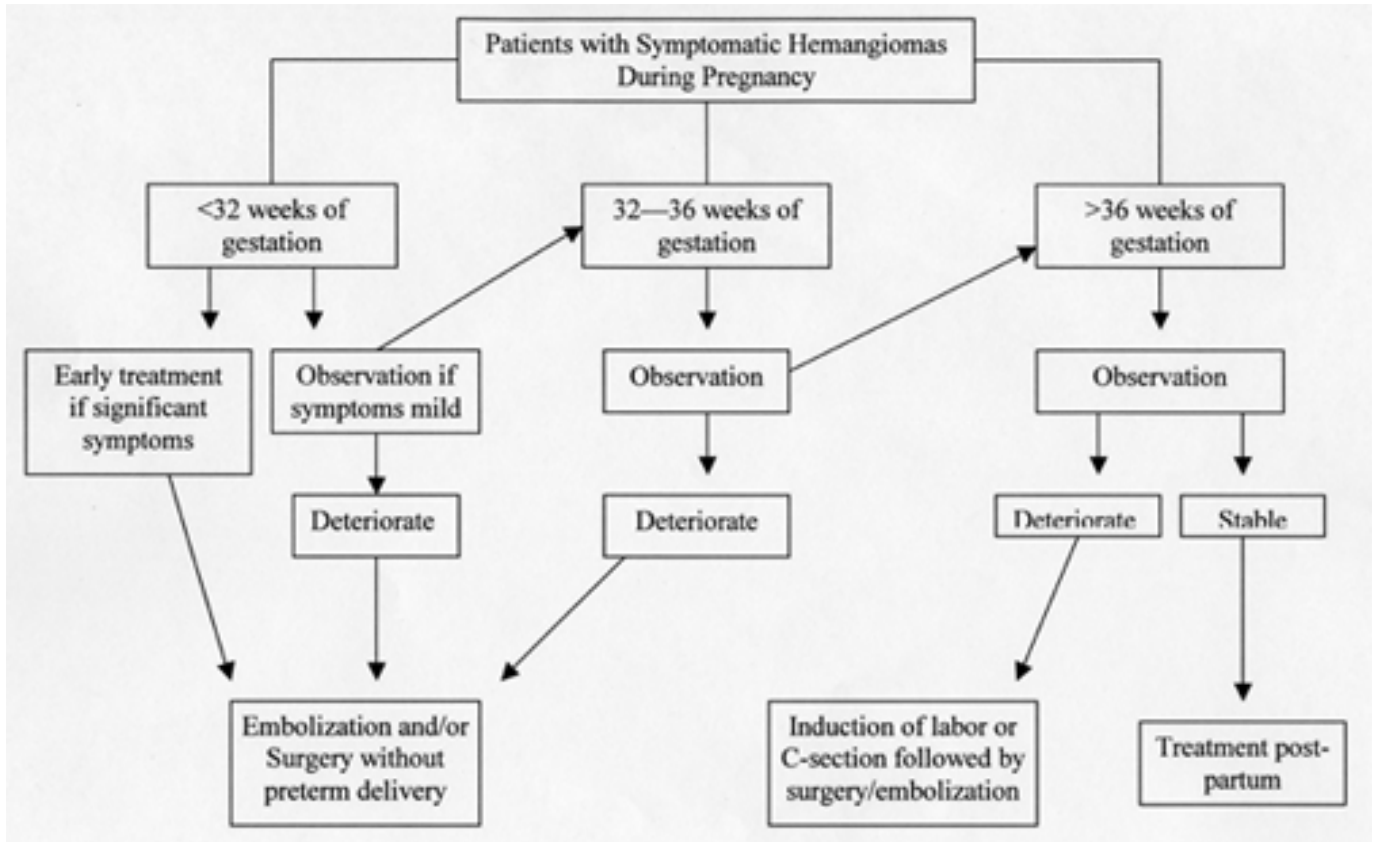

Fig. 4. Proposed treatment algorithm for symptomatic vertebral hemangiomas in pregnant women based on week of gestation. C-section = cesarean section. 
of the child and postpartum treatment. If neurological function deteriorates, one can consider induction of delivery or a cesarean section, followed by surgery with or without radiation, embolization, and/or sclerotherapy. Between 32 and 36 weeks of gestation, expectant observation may also be considered, although surgery should be reserved for severe cases of paraplegia with extensive spinal cord compression. One must consider the risk of preterm delivery if surgery is performed prepartum. For patients in whom gestation is earlier than 32 weeks, prepartum surgical treatment should be considered for those who are severely symptomatic. Surgery may be performed safely and will likely improve functional status, which plays an important role in maintaining a healthy pregnancy. If symptoms are mild or moderate, observation is warranted, but severe symptoms are likely to progress before delivery can be performed safely. There is limited experience with prepartum embolization, but it may be a useful technique that can alleviate or stabilize neurological symptoms, allowing for more time before delivery or surgery to optimize safety for the fetus. The risks to the fetus associated with radiation exposure during angiography and embolization must be considered; thus, prepartum embolization may not be suitable for all lesions.

It is important to understand that the overwhelming majority of patients documented in the literature recover well from pregnancy-related vertebral hemangioma, despite long periods of spastic paresis and weakness. According to our historical review, the morbidity and mortality rates after surgery for the pregnant patient and fetus are greater than the chance of permanent neurological deficit posttreatment. For any lesion presenting only with pain and paresthesias, we recommend observation alone. Also, patients being observed should be actively mobilized to prevent venous stasis and possible exacerbation of symptoms on bedrest. Although improvement in untreated patients after delivery has been reported, all patients treated prepartum, unless the lesion is gross totally resected, should be followed and adjuvant therapy considered after delivery because of the risk of recurrence with future pregnancies or with time.

\section{References}

1. Acquaviva R, Thevenot C: [Recurrent spinal cord compression caused by vertebral hemangioma; determinant role of pregnancy.] Maroc Med 36:942-944, 1957 (Fre)

2. Askenasy H, Behmoaram A: Neurological manifestations in haemangioma of the vertebrae. J Neurochem 20:276-284, 1957

3. Baker ND, Klein MJ, Greenspan A, et al: Symptomatic vertebral hemangiomas: a report of four cases. Skelet Radiol 15: 458-463, 1986

4. Bandiera S, Gasbarrini A, De Iure F, et al: Symptomatic vertebral hemangioma: the treatment of 23 cases and a review of the literature. Chir Organi Mov 87:1-15, 2002

5. Bouchez B, Gozet G, Le Coutour X, et al: [Medullary compression due to vertebral angioma in pregnancy. A case treated by embolization.] J Gynecol Obstet Biol Reprod (Paris) 13: 885-888, 1984 (Fre)

6. Bouchez B, Gozet G, Lecoutour X, et al: [Spinal cord compression caused by vertebral angioma during pregnancy. A case treated by embolization.] Presse Med 13:1696-1697, 1984 (Fre)

7. Bremnes RM, Hauge HN, Sagsveen R: Radiotherapy in the treatment of symptomatic vertebral hemangiomas: technical case report. Neurosurgery 39:1054-1058, 1996

8. Castel E, Lazennec JY, Chiras J, et al: Acute spinal cord com- pression due to intraspinal bleeding from a vertebral hemangioma: two case-reports. Eur Spine J 8:244-248, 1999

9. David M, Constans-Lamarch JP: Compression medullaire recidivante par hemangiome extradural. Role determinant des grossesses sur les rechutes. Rev Neurol (Paris) 87:638-644, 1952

10. Doppman JL, Oldfield EH, Heiss JD: Symptomatic vertebral hemangiomas: treatment by means of direct intralesional injection of ethanol. Radiology 214:341-348, 2000

11. Esparza J, Castro S, Portillo JM, et al: Vertebral hemangiomas: spinal angiography and preoperative embolization. Surg Neurol 10:171-173, 1978

12. Faria SL, Schlupp WR, Chiminazzo H Jr: Radiotherapy in the treatment of vertebral hemangiomas. Int J Radiat Oncol Biol Phys 11:387-390, 1985

13. Feydy A, Cognard C, Miaux Y, et al: Acrylic vertebroplasty in symptomatic cervical vertebral haemangiomas: report of 2 cases. Neuroradiology 38:389-391, 1996

14. Fields WS, Jones JR: Spinal epidural hemangioma in pregnancy. Neurology 7:825-828, 1957

15. Gabal AM: Percutaneous technique for sclerotherapy of vertebral hemangioma compressing spinal cord. Cardiovasc Intervent Radiol 25:494-500, 2002

16. Gangi A, Guth S, Imbert JP, et al: Percutaneous vertebroplasty: indications, technique, and results. Radiographics 23:e10, 2003

17. Gerszten PC, Ozhasoglu C, Burton SA, et al: CyberKnife frameless single-fraction stereotactic radiosurgery for benign tumors of the spine. Neurosurg Focus 14(5):E16, 2003

18. Goyal M, Mishra NK, Sharma A, et al: Alcohol ablation of symptomatic vertebral hemangiomas. AJNR Am J Neuroradiol 20:1091-1096, 1999

19. Guthkelch A: Hemangiomas involving the spinal epidural space. J Neurol Neurosurg Psychiatry 11:199-210, 1948

20. Jayakumar PN, Vasudev MK, Srikanth SG: Symptomatic vertebral haemangioma: endovascular treatment of 12 patients. Spinal Cord 35:624-628, 1997

21. Lam RL, Roulhac GE, Erwin HJ: Hemangioma of the spinal canal and pregnancy. J Neurosurg 8:668-671, 1951

22. Laredo JD, Reizine D, Bard M, et al: Vertebral hemangiomas: radiologic evaluation. Radiology 161:183-189, 1986

23. Lavi E, Jamieson DG, Granat M: Epidural haemangiomas during pregnancy. J Neurol Neurosurg Psychiatry 49:709-712, 1986

24. Liu CL, Yang DJ: Paraplegia due to vertebral hemangioma during pregnancy. A case report. Spine 13:107-108, 1988

25. Nelson DA: Spinal cord compression due to vertebral angiomas during pregnancy. Arch Neurol 11:408-413, 1964

26. Newman MJ: Spinal angioma with symptoms in pregnancy. J Neurochem 21:38-41, 1958

27. Nguyen JP, Djindjian M, Badiane S: [Vertebral hemangioma with neurologic signs. Clinical presentation, results of a survey by the French Society of Neurosurgery.] Neurochirurgie 35: 270-274, 305-308, 1989 (Fre)

28. Nguyen JP, Djindjian M, Pavlovitch JM, et al: Vertebral hemangioma with neurologic signs. Therapeutic results. Survey of the French Society of Neurosurgery. Neurochirurgie 35: 299-303, 305-308, 1989 (Fre)

29. Pastushyn AI, Slin'ko EI, Mirzoyeva GM: Vertebral hemangiomas: diagnosis, management, natural history and clinicopathological correlates in 86 patients. Surg Neurol 50:535-547, 1998

30. Pavlovitch JM, Nguyen JP, Djindjian M, et al: [Radiotherapy of vertebral hemangioma with neurologic complications.] Neurochirurgie 35:296-298, 305-308, 1989 (Fre)

31. Poungvarin N, Bhoopat W: Symptomatic vertebral haemangiomas: report of two cases. J Med Assoc Thai 74:363-368, 1991

32. Rami PM, McGraw JK, Heatwole EV, et al: Percutaneous vertebroplasty in the treatment of vertebral body compression fracture secondary to osteogenesis imperfecta. Skelet Radiol 31: $162-165,2002$ 


\section{Pregnancy-related vertebral hemangioma: management algorithm}

33. Redekop GJ, Del Maestro RF: Vertebral hemangioma causing spinal cord compression during pregnancy. Surg Neurol 38: 210-215, 1992

34. Schwartz DA, Nair S, Hershey B, et al: Vertebral arch hemangioma producing spinal cord compression in pregnancy. Diagnosis by magnetic resonance imaging. Spine 14:888-890, 1989

35. Schwartz TH, Hibshoosh H, Riedel CJ: Estrogen and progesterone receptor-negative T11 vertebral hemangioma presenting as a postpartum compression fracture: case report and management. Neurosurgery 46:218-221, 2000

36. Shapiro GS, Millett PJ, DiCarlo EF, et al: Spinal epidural hemangioma related to pregnancy. Skelet Radiol 30:290-294, 2001
37. Tekkok IH, Acikgoz B, Saglam S, et al: Vertebral hemangioma symptomatic during pregnancy - report of a case and review of the literature. Neurosurgery 32:302-306, 1993

38. Templin CR, Stambough JB, Stambough JL: Acute spinal cord compression caused by vertebral hemangioma. Spine J 4: 595-600, 2004

Manuscript received April 25, 2005.

Accepted in final form June 14, 2005.

Address reprint requests to: John H. Chi, M.D., M.P.H., 505 Parnassus Avenue, M 779, San Francisco, California 94143-0112. email: chijo@neurosurg.ucsf.edu. 